\title{
РАЗРАБОТКА И ИССЛЕДОВАНИЕ НОВОГО СПОСОБА ГАЗООЧИСТКИ ОТ ЧАСТИЦ РАЗМЕРОМ МЕНЕЕ 2,5 МКМ
}

\author{
Хмелев Владимир Николаевич1, \\ vnh@bti.secna.ru
}

\author{
Шалунов Андрей Викторович 1 , \\ shalunov@bti.secna.ru
}
Боченков Александр Сергеевич1, sanya.bochankov@mail.ru

\author{
Нестеров Виктор Александрович', \\ nva@u-sonic.ru
1 Бийский технологический институт (филиал)
«Алтайского государственного технического университета им. И.И. Ползунова», Россия, 659305, г. Бийск, ул. имени Героя Советского Союза Трофимова, 27.

\begin{abstract}
Актуальность исследования обусловлена отсутствием эфффективных методов и средств борьбы с загрязнением атмосфреры субмикронными частицами размером менее 2,5 мкм. Применяемое для этих целей ультразвуковое воздействие не позволяет обеспечить степень укрупнения дисперсных частии, достаточную для их осаждения или улавливания существующим газоочистным оборудованием. Как показывают экспериментальные исследования, даже при максимальном уровне звукового давления (при превышении которого инициируется обратный процесс - диспергирование капель или разрушении агломератов частии) эфффективность ультразвуковой коагуляции оказывается недостаточной, особенно при малой счетной концентрации частиц. Это обуславливает необходимость выявление новых фризических эфффектов и разработки новых способов воздействия, повышающих эффрективность коагуляции тонкодисперсных частиц.

Цель: разработка способа для повышения эфффективности ультразвуковой коагуляции частии размером менее 2,5 мкм за счет формирования вихревых акустических течений в тонком воздушном промежутке между излучателем и отражателем и создание устройства для его практической реализации.

Результаты. Предложен новьй способ и устройство для очистки газов от дисперсных частиц размером менее 2,5 мкм за счет увеличения времени ультразвукового воздействия на каждую частичку и создания зон локального увеличения концентрации субмикронных частии. Это обеспечивается за счет формирования вихревых потоков между излучающей (в виде изгибно-колеблющегося диска) и отражающей поверхностями. Локальная зона повышенной концентрации частиц формируется в перифрерийной области вихря за счет дрейфра частиц под действием центробежных сил из центральной области вихря к его периферии. Предложенный способ обеспечивает повышение эфффективности с 13 до 50 \%, в зависимости от исходной концентрации дисперсных частии.
\end{abstract}

\section{Ключевые слова: \\ Ультразвук, ультразвуковая коагуляция, излучатель, вихревые потоки, аэрозоль.}

\section{Введение}

В последнее время большое внимание уделяется повышению эффективности способов газоочистки, особенно от частиц размером менее 2,5 мкм, обладающих высокой общей поверхностью (55\% и более от общей поверхности частиц) и счетной концентрацией (95\% и более от суммарной счётной концентрации содержащихся в атмосфере). Из-за малых размеров подобные частицы, задерживаясь в воздухе долгое время, оказывают негативное влияние на здоровье человека.

Реализуемые в настоящее время способы коагуляции основаны на различных физических явлениях и принципах воздействия на загрязняющие газ частицы, например, на процессах инерции и центробежных явлениях [1]. Эффективность практической реализации этих способов коагуляции может достигать 70-90\% для частиц размером более 5-10 мкм. На меньшие по размерам частицы воздействие практически не оказывается. Принято считать, что обусловлено это отсутствием эффективных физических механизмов воздействия на меньшие по размерам (в том числе субмикронные) частицы.
Поскольку звуковые колебания высокой интенсивности крайне опасны для окружающего мира, возникает необходимость использовать ультразвуковые колебания ( $\mathrm{f}>20$ кГц). УЗ воздействие абсолютно безопасно для окружающих даже при высокой силе ультразвука (J>150 дБ). Поэтому наиболее эффективные способы удаления субмикронных частиц основаны на применении ультразвуковых (УЗ) колебаний высокой интенсивности, которые способны осуществлять дополнительное воздействие и на газовую среду, и на частицы в ней. При этом воздействие УЗ колебаниями на газовые среды создаёт два типа нелинейных эффектов $[2,3]$, влияющих на дисперсные частицы в газах:

- эффекты, возникающие за счёт явлений переноса импульса газовой фазы между частицами;

- эффекты, возникающие за счёт колебательных движений твердых частиц друг относительно друга. Первая группа эффектов обуславливает гидродинамический механизм (впервые описанный В. Кёнигом в 1891 г.) агломерации частиц, когда эквивалентные силы гидродинамического взаимодействия (переносчиками взаимодействия выступают молекулы 
сплошной газовой фазы) приближают частицы друг к другу и способствуют их агломерации. При наложении УЗ колебаний отдельные частицы подвергаются воздействию колебаний и потоков окружающего газа, начинают двигаться быстрее, вероятность их столкновений с другими частицами возрастает, они чаще соударяются и объединяются. Происходит увеличение их размеров, вследствие чего становится возможным дальнейшее улавливание укрупненных частиц при помощи различных известных способов, реализуемых, например, с помощью сепараторов [4-8].

Однако при очистке газов от частиц размером менее 2,5 мкм данная сила взаимодействия оказывается малой, поскольку она фактически пропорциональна 6-й степени диаметра частиц. Поэтому, например, для частиц размером 2,5 мкм эта сила в 64 раза меньше, чем для частиц размером 5 мкм. Кроме того, при малых концентрациях частиц существенно уменьшает силу взаимодействия большое расстояние между частицами.

В свою очередь, вторая группа эффектов реализуется в ортокинетическом механизме (впервые описанным Брандтом, Х. Фройндом, Е. Хидеманом), заключающемся в том, что частица колеблется с газовой фазой не синхронно, а осуществляет скольжение относительно газовой фазы и колеблется с меньшей амплитудой смещения, чем сам газ. Эффект скольжения связан с инерционностью частицы. Для частиц тонкодисперсных размером менее 2,5 мкм модуль коэффициента увлечения близок к 1 , т. е. все частицы колеблются практически одинаково, и соответственно ортокинетическое взаимодействие также не реализуется $[4,7]$.

Поэтому даже способы коагуляции, основанные на применении ультразвукового воздействия, теоретически являются малоэффективными, и по данным многочисленных исследований [4, 9-12] эффективность способов УЗ коагуляции частиц размером 2,5 мкм и менее не превышает 20-35\%.

К сожалению, предельные возможности ультразвукового воздействия при коагуляции тонкодисперсных частиц (2,5 мкм и менее) до настоящего времени экспериментально не определены. Это не позволяет реализовывать практические конструкции ультразвуковых коагуляторов с максимальной эффективностью и, главное, не дает возможности выявлять пути решения проблемы дальнейшего повышения эффективности УЗ коагуляции мелких частиц размером 2,5 мкм и менее.

Поэтому появляется необходимость проведения исследований для выявления предельных возможностей ультразвукового воздействия и выявления путей дальнейшего развития ультразвуковой коагуляции тонкодисперсных частиц

\section{Стенд для исследования ультразвуковой коагуляции субмикронных частиц}

Для проведения экспериментов был предложен и разработан специализированный стенд для измерения эффективности ультразвуковой коагуляции. Основное требование к создаваемому стенду - обеспечение максимально возможного (предельного) УЗ воздействия на очищаемый газ. Очевидно, что необходимым и достаточным должно стать УЗ воздействие с силой звука 160 дБ (интенсивностью 1 Вт/см²). Воздействие с большей силой звука будет осуществлять не только коагулирующее воздействие, но и разрушать объединения (конгломераты) частиц или просто распылять жидкие частицы [9-11].

В качестве коагулируемых частиц было принято решение использовать жидкие частицы калиброванного размера, создаваемые при помощи специального устройства - компрессорного генератора Topas ATM 226 с регулируемым объемом потока аэрозолей. Генератор Topas ATM 226 обеспечивает формирование аэрозоля со стабильными во времени дисперсными характеристиками: размеры формируемых частиц в интервале $0,1-2,5$ мкм и объемное содержание $10^{8}$ частиц/см ${ }^{3}$. В качестве распыляемой жидкости применена известная аэрозольная жидкость DEHS.

Конструктивное исполнение стенда показано на рис. 1. Представленный стенд реализует различные условия воздействия УЗ колебаниями, а также позволяет измерять эффективность ультразвуковой коагуляции по отношению оптической плотности аэрозоля на выходе и входе коагуляционной камеры. Стенд включает в себя следующие составные блоки:

- когуляционная камера;

- ультразвуковая колебательная система (УЗКС) в комплекте с излучателем ультразвуковых колебаний;

- генератор колебаний ультразвуковой частоты, предназначенный для питания УЗКС и позволяющий формировать уровень звукового давления в камере 130-160 дБ;

- генератор тонкодисперсных аэрозолей, способный обеспечить формирование мелких капель размером до 1 мкм;

- блок оптического измерительного устройства; Коагуляционная камера включает:

- пространство, в котором происходит воздействие на формируемый аэрозоль колебаниями ультразвуковой частоты с прозрачными боковыми стенками;

- входной патрубок, соединенный с генератором аэрозолей;

- выходной патрубок с установленным вентилятором для обеспечения транспортирования аэрозоля через коагуляционную камеру. Вентилятор обеспечивает скорость потока равную 0,85 м/c;

- на входном и выходном патрубках предусмотрены расположенные друг против друга отверстия для установки оптических пар: инфракрасный светодиод - фотоприемник, обеспечивающие измерение оптической плотности аэрозоля.

Для обеспечения максимального ультразвукового воздействия в предложенном стенде был реализован режим резонансного усиления колебаний в коагуляционной камере при помощи круглого металлического отражателя. Для обеспечения резонансных режимов и установления различных уровней ультразвуко- 
вого давления в стенде была реализована возможность регулировать расстояние между излучателем и отражателем путем перемещения излучателя вдоль вертикальной оси коагуляционной камеры $[12,13]$.
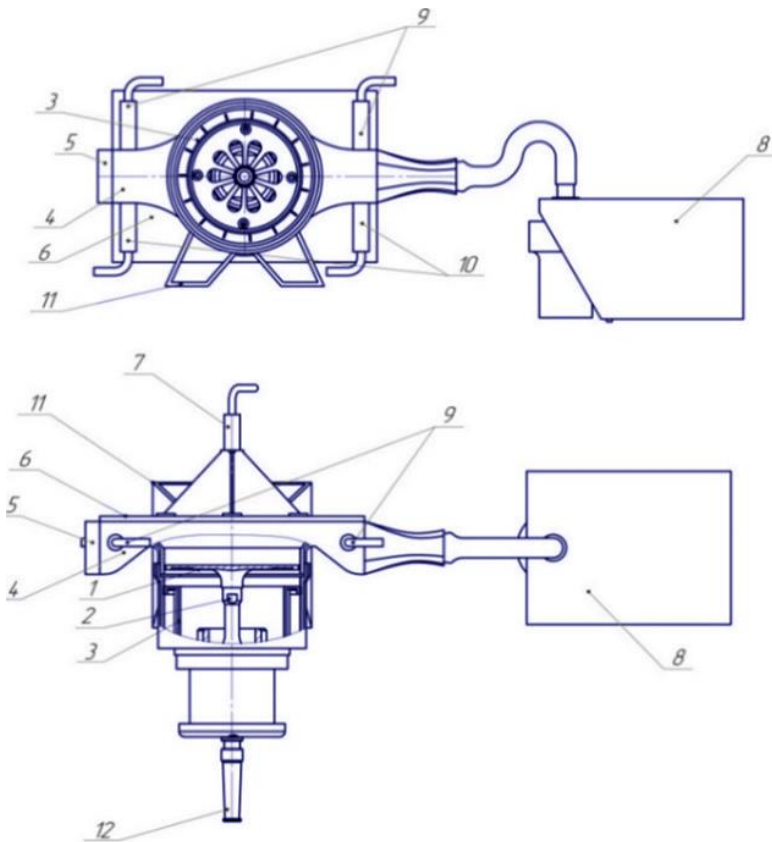

Pис. 1. Конструкция экспериментального стенда: 1) ультразвуковой излучатель; 2) пьезоэлектрическая ультразвуковая колебательная система; 3) фланеи; 4) коагуляиионная камера; 5) вентилятор; 6) отражательная пластина; 7) микрофон; 8) генератор газодисперсной взвеси (аэрозоля); 9) фотодиоды выходного и входного каналов, работающие в инфракрасном диапазоне; 10) светодиоды выходного и входного каналов, работаюшие в инфракрасном диапазоне; 11) опорная рама стенда; 12) кабель питания излучателя ультразвуковых колебаний

Fig 1. Design of the experimental stand: 1) ultrasonic radiator; 2) piezoelectric ultrasonic oscillating system (UPOS); 3) UPOS flange; 4) coagulation chamber; 5) fan; 6) transparent reflector; 7) microphone; 8) gaseous suspension (aerosol) generator; 9) infrared photodiodes of input and output channels; 10) infrared LEDs for input and output channels; 11) stand support; 12) cable for power supply of the ultrasonic radiator

Как следует из описания стенда, его основу составляет источник УЗ воздействия - ультразвуковая колебательная система со специальными излучателями.

\section{Устройство создания УЗ колебаний}

\section{с продольно-колеблющимся излучателем}

Для определения предельных возможностей УЗ воздействия при коагуляции тонкодисперсных аэрозолей в режиме плоской стоячей волны (наиболее частот встречающемся на практике) была разработана УЗКС с продольно-колеблющимся излучателем. Основой конструкции данного излучателя служит тело цилиндрической формы резонансной (полуволновой) длины, соединенное с электроакустическим преобра- зователем. Эскиз УЗКС вместе с поршневым продольно-колеблющимся излучателем представлен на рис. $2[13,14]$.

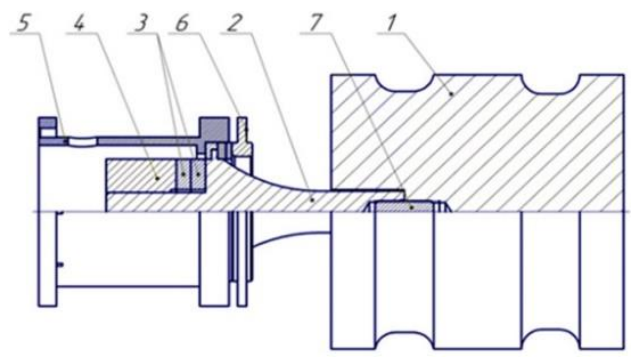

Pис. 2. Эскиз УЗКС с поршневым излучателем: 1) излучатель УЗ колебаний; 2) концентраторусилитель механических колебаний; 3) пьезокерамические элементы; 4) отражающая накладка; 5) корпус УЗКС; 6) фланец; 7) шпилька

Fig. 2. Sketch of an ultrasonic vibrating system with a piston-type radiator: 1) ultrasonic radiator; 2) concentrator of the piezoelectric transducer; 3) piezoceramic elements; 4) reflective pad; 5) body; 6) flange; 7) hairpin

Для обеспечения максимальной равномерности колебаний торцевой поверхности было предложено выполнить кольцевые проточки [12-14].

На рис. 3 представлена фотография УЗКС с излучателем поршневого типа и форма колебаний излучателя.

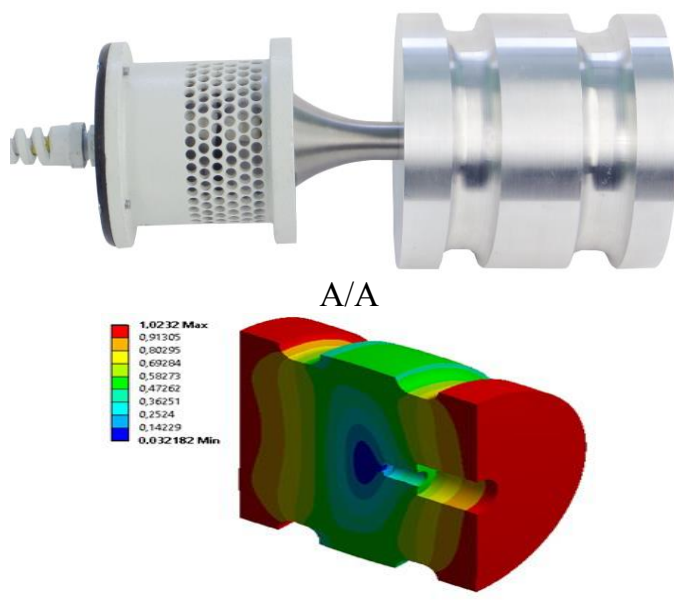

Б/B

Pис. 3. Фотография (А) и распределение колебаний УЗ излучателя поршневого типа (Б)

Fig. 3. Photo of the piston-type radiator (A); oscillation mode of piston-type ultrasonic radiator (Б)

В табл. 1 представлены основные технические характеристики ультразвукового излучателя поршневого типа.

Для питания созданного излучателя был использован ультразвуковой генератор (рис. 4), позволяющий создать, совместно с ультразвуковым излучателем, внутри коагуляционной камеры уровень звукового давления 130-160 дБ. Генератор позволяет устанавливать и контролировать время и уровень мощности ультразвукового воздействия [15]. 
Таблица 1. Технические характеристики ультразвукового излучателя поршневого типа

Table 1. Technical characteristics of piston-type radiator

\begin{tabular}{|l|c|}
\hline \multicolumn{1}{|c|}{ Параметр/Рarameter } & \multicolumn{1}{|c|}{ Значение/Value } \\
\hline $\begin{array}{l}\text { Тип излучающего элемента } \\
\text { Radiating element type }\end{array}$ & $\begin{array}{c}\text { Поршневой продольно- } \\
\text { колеблющийся } \\
\text { Piston-type longitudinally oscillating }\end{array}$ \\
\hline $\begin{array}{l}\text { Диаметр поверхности излуча- } \\
\text { теля, мм } \\
\text { Radiating surface diameter, mm }\end{array}$ & 104 \\
\hline $\begin{array}{l}\text { Рабочая частота, кГц } \\
\text { Operation frequency, kHz }\end{array}$ & 23,5 \\
\hline $\begin{array}{l}\text { Максимальная потребляемая } \\
\text { электрическая мощность, Вт } \\
\text { Еlectric роwer, W }\end{array}$ & \\
\hline $\begin{array}{l}\text { Уровень звукового давления } \\
\text { на дистанции 1 м, не менее дБ } \\
\begin{array}{l}\text { Sound pressure level ат а } \\
\text { distance of 1 m, dВ }\end{array}\end{array}$ \\
\hline
\end{tabular}

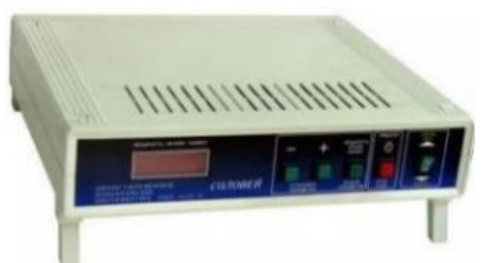

Puс. 4. Ультразвуковой генератор

Fig. 4. Ultrasonic generator

Представленный ультразвуковой излучатель способен обеспечить предельно возможное воздействие для коагуляции тонкодисперсных и субмикронных частиц в газовых потоках.

\section{Устройство для измерения}

\section{оптической плотности аэрозоля}

Устройство основано на измерении оптической плотности очищаемого газа.

Система определения оптической плотности аэрозоля реализована на микроконтроллере семейства AVR. Структурная схема измерительного устройства представлена на рис. 5.

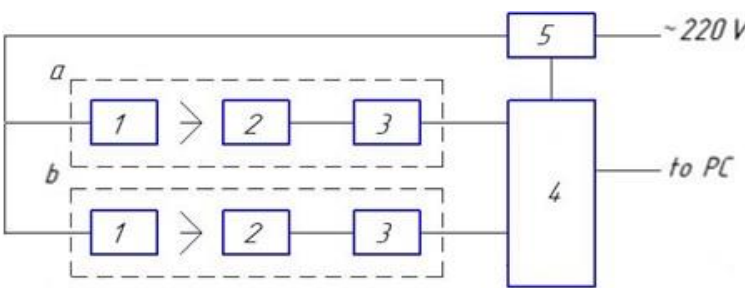

Pис. 5. Блок-схема устройства для измерения оптической плотности аэрозоля: 1) светодиод, работающий в инфракрасном диапазоне; 2) фотодиод, работающий в инфракрасном диапазоне; 3) усилитель сигнала с фотодиода; 4) микроконтроллер; 5) блок питания; $a$, b) входной и выходной каналь для измерения

Fig. 5. Block-diagram of a device for measuring the optical density of an aerosol: 1) infrared LED;2) infrared photodiode; 3) amplifier photodiode signal; 4) microcontroller; 5) power supply; $a, b$ ) input and output channels for measurement
Оптическая плотность определяется по отношению электрических сигналов на выходах инфракрасных фотодиодов, установленных на выходе и входе из камеры.

Измерение оптической плотности аэрозоля осуществляется следующим образом. Световой поток со светодиодов, работающих в инфракрасном диапазоне, проходит через входной (рис. $5, a$ ) и, соответственно, выходной (рис. 5, $b$ ) патрубок коагуляционной камеры, заполненные аэрозолем.

Световой поток частично рассеивается на каплях аэрозоля, а оставшаяся его часть попадает на блок преобразования и усиления измерительного сигнала, который представлен операционным усилителем AD712JNZ и инфракрасным фотодиодом. Полученный сигнал, пропорциональный оптической плотности аэрозоля, поступает на аналогово-цифровой преобразователь микроконтроллера AVR Atmega48. По запросу измерительное устройство передает полученные данные на персональный компьютер через последовательный порт RS-232.

Перед проведением измерений выполнялась калибровка измерительного устройства посредством обеспечения равенства снимаемых с фотодиодов на входе и выходе сигналов при отсутствии аэрозоля. На основе отношения значений оптической плотности аэрозоля на выходе и входе коагуляционной камеры рассчитывалась эффективность процесса коагуляции по следующей формуле:

$$
\delta=\left(1-\frac{A_{\mathrm{Bbx}}}{A_{\mathrm{Bx}}}\right) \cdot 100 \%,
$$

где $A_{\text {вых }}$ и $A_{\text {вх }}$ - измеренные значения сигнала на фотодиодах, установленных на выходе и входе камеры, соответственно.

Величины $A_{\text {вых }}$ и $A_{\text {вх }}$ определялись по формуле:

$$
A=\frac{\sum_{j=1}^{n} A_{j}}{n}-A_{0},
$$

где $A_{0}$ - величина выходного сигнала, соответствующая состоянию воздушной среды, свободной от дисперсных примесей (аэрозоля); $A_{j}$ - отдельный элемент в измерительной последовательности; $n$ - количество элементов измерительной последовательности, равное $10 ; j$ - номер элемента [12-14].

Кроме того, в процессе УЗ воздействия на аэрозоль в коагуляционной камере осуществлялось визуальное наблюдение за движением аэрозольных частиц через прозрачные боковые стенки для выявления возможных неоднородностей и потоков. Фиксация осуществлялась при помощи цифровой фотокамеры. Для поршневого излучателя формирования акустических потоков не наблюдалось.

\section{Определение уровня звукового давления} и эффективности поршневого излучателя

Уровень звукового давления определялся в тонком промежутке при различных расстояниях между по- 
верхностями поршневого излучателя и отражателя. Измерения проводились шумомером Экофизика-110А (способен работать в ультразвуковом диапазоне частот до 40 кГц). Микрофон шумомера размещался по центру отражателя. При измерении уровня звукового давления расстояние между излучателем и отражателем дискретно изменялось с шагом 1 мм за счет перемещения ультразвукового излучателя с пьезоэлектрической ультразвуковой колебательной системой (рис. 1, поз. 1, 2 , соответственно). В каждой точке уровень звукового давления измерялся в течение 1 минуты, после чего результаты измерений усреднялись. Во время измерения уровня звукового давления подача аэрозоля в коагуляционную камеру не осуществлялась.

Зависимость уровня звукового давления от расстояния между поверхностями поршневого излучателя и отражателя представлена в виде графика (рис. 6).

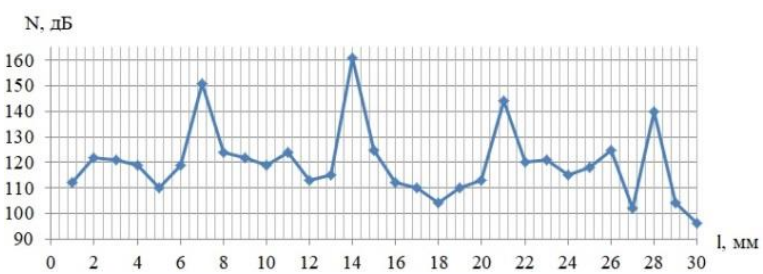

Pис. 6. График уровня звукового давления в зависимости от отношения расстояния между поверхностями поршневого продольно-колеблющего излучателя и отражателя

Fig. 6. Graph of the sound pressure level depending on the ratio of the distance between the surfaces of the piston longitudinally oscillating radiator and the reflector

Выраженные колебания, наблюдаемые на графике на рис. 6, вызваны образованием резонансных явлений в тонком воздушном промежутке между поверхностью излучателя и отражателя.

Из результатов измерений следует, что используемый излучатель на резонансном промежутке, равном одной длине волны, формирует максимальный уровень звукового давления 160 дБ [7, 16-18].

Результаты исследования эффективности процесса ультразвуковой коагуляции для поршневого продольно-колеблющегося излучателя относительно расстояния до поверхности отражателя представлены на гистограмме на рис. 7.

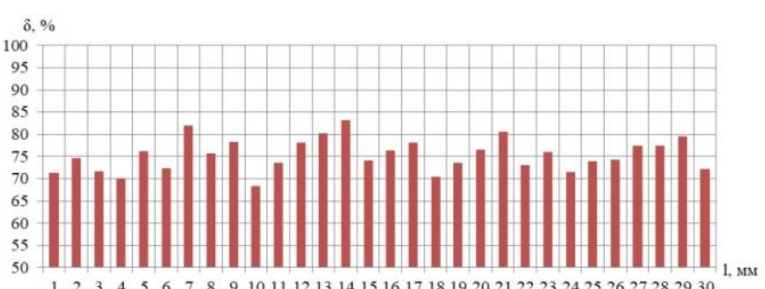

Рис. 7. Гистограмма эффективности коагуляциии в зависимости от расстояния между поверхностью поршневого продольно-колеблющуегося излучателя и поверхностью отражателя

Fig. 7. Bar chart of coagulation efficiency as a function of the distance between the surface of the piston longitudinally oscillating radiator and the surface of the reflector
Из анализа гистограммы следует, что пик эффективности коагуляции приходится на расстояние одной длины волны ультразвуковых колебаний (14 мм). На этом расстоянии эффективность коагуляции достигает своего максимума.

На рис. 8 представлено отношение эффективности коагуляции к уровню звукового давления, полученного на резонансном расстоянии между излучателем поршневого типа и отражателем равным одной длине волны.

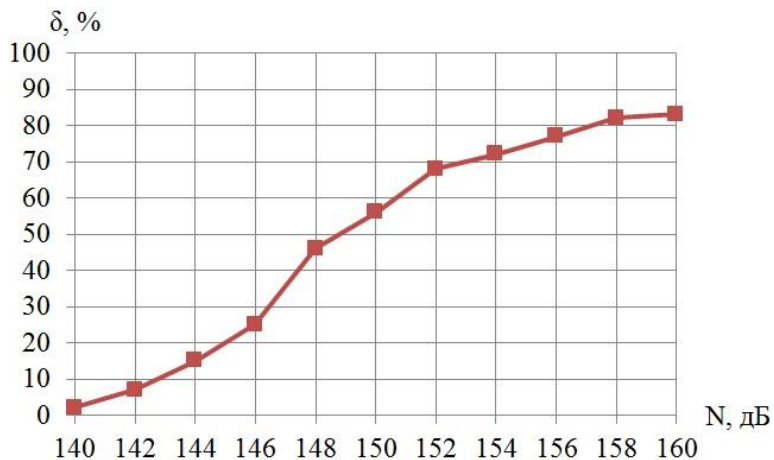

Pис. 8. Отношение эффективности ультразвуковой коагуляции к уровню звукового давления для пориневого излучателя

Fig. 8. Dependence of the efficiency of ultrasonic coagulation on the level of sound pressure for a piston-type radiator

Анализ зависимости на рис. 8 позволил установить, что эффективность коагуляции с использованием излучателя поршневого типа достигает своего максимума, равного 83 \%, при уровне звукового давления в 160 дБ. Это значение следует считать предельной эффективностью ультразвуковой коагуляции частиц размером 2,5 мкм в максимально возможном по силе ультразвукового воздействия поле механических колебаний, создаваемых излучателем поршневого типа.

Далее были проведены эксперименты по исследованию эффективности процесса коагуляции при помощи поршневого излучателя при различных концентрациях аэрозоля. Результаты эксперимента представлены на рис. 9.

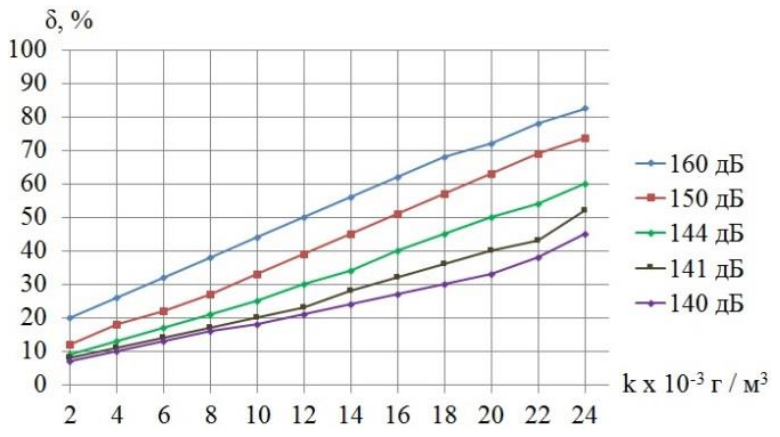

Pис. 9. Зависимость эффективности коагуляции от начальной концентрацчи аэрозоля для излучателя поршневого типа

Fig. 9. Dependence of the coagulation efficiency on the initial concentration for a piston-type radiator 
Как следует из представленных зависимостей, эффективность коагуляции существенным образом зависит от начальной концентрации аэрозоля. При ма-

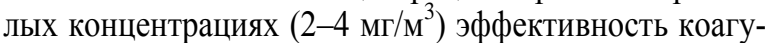
ляции в режиме плоской стоячей волны (формируемой плоским излучателем) не превышает $30 \%$, что делает нецелесообразным ее применение.

Таким образом, проведенные исследования для случая УЗ воздействия в режиме плоской стоячей волны позволили установить предельные возможности ультразвуковой коагуляции частиц размером 2,5 мкм и менее, т. е. установить значение предельной эффективности ультразвуковой коагуляции, равное 83 \%.

\section{Создание нового способа УЗ коагуляции}

Очевидно, что повысить эффективность коагуляции субмикронных частиц можно за счет повышения времени УЗ воздействия на каждую частичку и создания зон локального увеличения концентрации тонкодисперсных и субмикронных частиц. Практически это может быть обеспечено путем формирования вихревых потоков между излучающей и отражающей поверхностями. Такие потоки должны обеспечить повышение эффективности ультразвуковой коагуляции частиц размером менее 2,5 мкм. Поэтому для проведения дальнейших исследований предпринята попытка создания иных условий в ультразвуковых полях, формируемых при помощи других известных типов ультразвуковых излучателей (способных создавать аналогичные по силе звука воздействия).

\section{Устройство создания УЗ колебаний с изгибно- колеблющимся излучателем}

Излучатель был выполнен в виде изгибноколеблющегося диска с центральным возбуждением от пьезоэлектрической колебательной системы. Ультразвуковые колебания, как правило, возбуждаются на кратных основной моде колебаний $(3,5,7$ и т. д.).

На рис. 10 представлен эскиз УЗКС с излучателем в виде изгибно-колеблющейся пластины (диска) [12, 13].

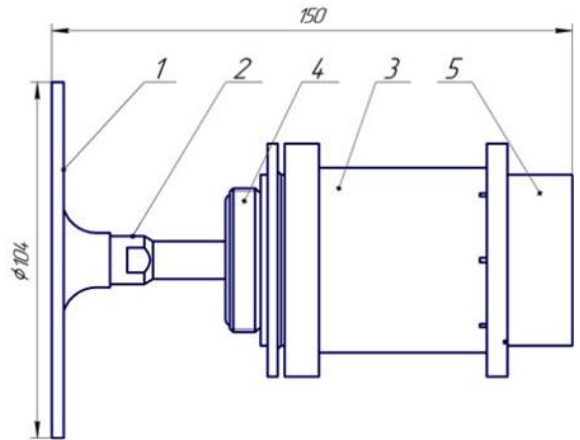

Рис. 10. Эскиз УЗКС с излучателем в виде плоского диска: 1) дисковый излучатель; 2) пьезоэлектри ческий преобразователь; 3) корпус УЗКС 4) фланеи; 5) вентилятор

Fig. 10. Sketch of an ultrasonic oscillating system with a radiator in the form of a flexural-oscillating flat disk: 1) disk radiator; 2) piezoelectric transducer; 3) body; 4) flange; 5) fan
На рис. 11 представлена фотография УЗКС с дисковым излучателем и форма колебаний дискового излучающего элемента.

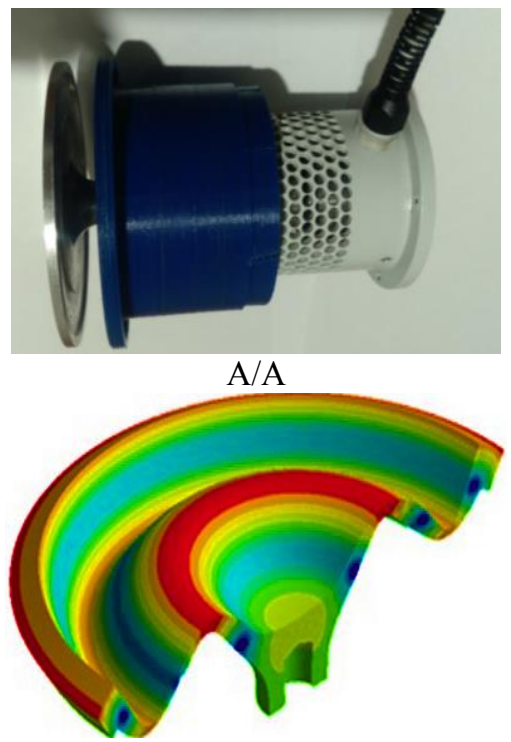

Б/B

Puc. 11. Фотография изгибно-колеблющегося дискового излучателя (A); форма колебаний дискового излучающего элемента (Б)

Fig. 11. Photo of a flexural-oscillating disk radiator (A); oscillation mode of disk radiator $(B)$

В табл. 2 представлены основные технические характеристики изгибно-колеблющегося дискового излучателя.

Таблица 2. Технические характеристики изгибноколеблющегося дискового излучателя для газовых сред

Table 2. Technical characteristics of flexural-oscillating disk radiator for gas environments

\begin{tabular}{|c|c|}
\hline Параметр/Parameter & Значение/Value \\
\hline $\begin{array}{l}\text { Тип излучающего элемента } \\
\text { Radiating element type }\end{array}$ & $\begin{array}{c}\text { Дисковый изгибно- } \\
\text { колеблющийся } \\
\text { Disk flexural-oscillation }\end{array}$ \\
\hline $\begin{array}{l}\text { Диаметр излучающей поверхно- } \\
\text { сти, мм } \\
\text { Radiating surface diameter, mm }\end{array}$ & 104 \\
\hline $\begin{array}{l}\text { Рабочая частота, кГц } \\
\text { Operation frequency, } \mathrm{kHz}\end{array}$ & 23,5 \\
\hline $\begin{array}{l}\text { Максимальная потребляемая } \\
\text { электрическая мощность, Вт } \\
\text { Electric power, W }\end{array}$ & 60 \\
\hline $\begin{array}{l}\text { Уровень звукового давления на } \\
\text { дистанции } 1 \text { м, не менее дБ } \\
\text { Sound pressure level at a distance } \\
\text { of } 1 \mathrm{~m}, \mathrm{~dB}\end{array}$ & 145 \\
\hline
\end{tabular}

Как следует из представленных технических характеристик, такой ультразвуковой излучатель в виде плоского диска способен обеспечить необходимое ультразвуковое воздействие (аналогичное рассмотренному ранее) для реализации процесса ультразвуковой коагуляции тонкодисперсных частиц в газовых средах $[11,14]$. 


\section{Визуальное наблюдение формирования} акустических потоков

В процессе воздействия на аэрозоль в коагуляционной камере было установлено, что визуально можно наблюдать формирование вихревых акустических течений между дисковым излучателем и отражателем (рис. 12).

Для дискового излучателя вихревой поток заполняет весь промежуток между излучателем и отражателем. При этом на отражателе наблюдается осаждение капель, формирующих кольцевой рисунок (рис. 13).
При расстоянии между излучателем и отражателем равном $\lambda / 4$ осажденные на поверхности отражателя капли (образуются в результате ультразвуковой коагуляции аэрозоля) образуют концентрические кольца. При этом внутренние кольца имеют более правильную форму и более четкую границу, чем внешние кольца. При расстоянии 7 и 14 мм структура осаждений также является регулярной, однако представляет собой сочетание пятен, равномерно покрытых осажденными каплями, и концентрических колец При этом внешние кольца не замкнутые. Это, повидимому, связано со структурой акустического поля в зазоре и формируемыми акустическими течениями.

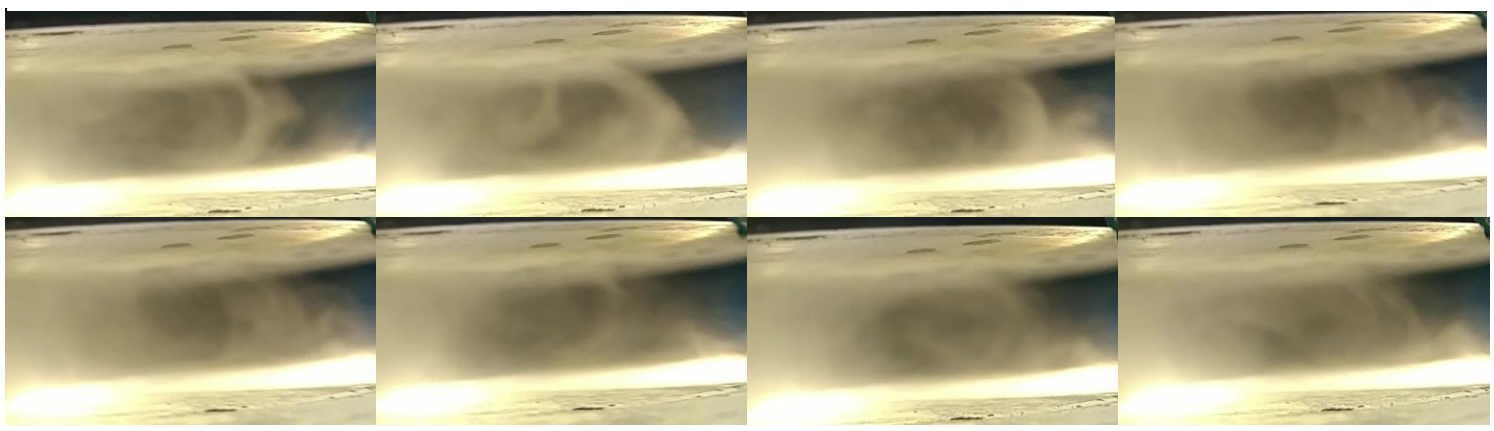

Pис. 12. Формируемые акустические потоки вихревого типа дискового излучателя

Fig. 12. Formed acoustic flows of vortex-type disk radiator

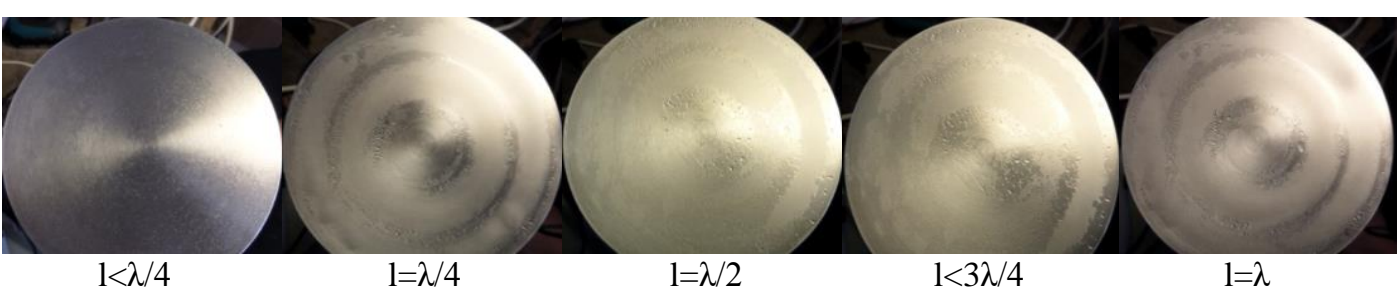

Рис. 13. Структуры, формируемые на отражателе осаждаемыми каплями

Fig. 13. Structures formed on a reflector by deposited drops

При расстояниях меньше 4 мм $(\lambda / 4)$ и больше 14 мм $(\lambda)$ формирование вихревых потоков не происходит. При этом на расстояниях, превышающих 14 мм, наблюдается значительная турбулизация газокапельной взвеси, приводящая к снижению эффективности коагуляции аэрозоля. На отражателе при этом наблюдается хаотичное осаждение крупных капель аэрозоля диаметром 15-100 мкм. То есть ускоренного осаждения высокодисперсных частиц на отражателе не наблюдается.

Следовательно, при анализе имеющихся данных можно сделать вывод о факте возникновения в тонком промежутке между излучателем дискового типа и отражателем на определенных расстояниях вихревых акустических течений. Дальнейшие исследования были посвящены изучению влияния акустических вихрей на эффективность ультразвуковой коагуляции.

Определение уровня звукового давления и эфффективности изгибно-колеблющегося дискового излучателя

По аналогии с поршневым продольно-колеблющимся излучателем в виде графика представлены ре- зультаты измерения уровня звукового давления для дискового изгибно-колеблющегося излучателя относительно расстояния до отражателя (рис. 14).

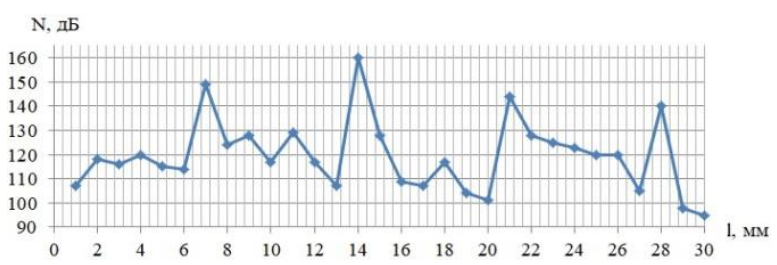

Рис. 14. График уровня звукового давления в зависимости от отношения расстояния между поверхностями дискового излучателя и отражателя

Fig. 14. Graph of the sound pressure level depending on the ratio of the distance between the surfaces the disk radiator and the reflector

При рассмотрении графика на рис. 14 можно сделать вывод, что аналогично поршневому продольноколеблющемуся излучателю на резонансном промежутке, равном одной длине волны, формируется уровень звукового давления 160 дБ [8, 16-19]. 
При этом относительное отличие уровней звукового давления, формируемых дисковым и поршневым излучателями, при одинаковом расстоянии от отражателя не превышает \pm 2 дБ во всем диапазоне расстояний. Следовательно, условия проведения экспериментальных исследований для обоих типов излучателей были одинаковы.

Равенство уровней звукового давления, формируемого излучателями обоих типов, позволяет провести сравнение эффективности коагуляции, обеспечиваемой излучателем, и определить влияние вихревых потоков, создаваемых изгибно-колеблющимся излучателем, на эффективность коагуляции.

Результаты эффективности коагуляции с использованием дискового излучателя представлены на рис. 15.

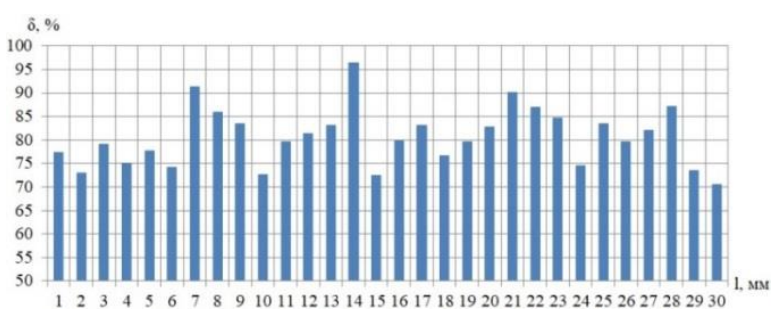

Pис. 15. Гистограмма эффективности коагуляции в зависимости от расстояния между поверхностью дискового излучателя и поверхностью отражателя

Fig. 15. Bar chart of coagulation efficiency as a function of the distance between the surface of the disk radiator and the surface of the reflector

Из сравнения гистограмм на рис. 7 и 15 следует, что для обоих излучателей пик эффективности коагуляции приходится на расстояние одной длины волны ультразвуковых колебаний. На этом расстоянии эффективность коагуляции достигает своего максимума.

На рис. 16 представлена зависимость эффективности коагуляции от уровня звукового давления, полученная на резонансном расстоянии одной длины волны между поверхностями дискового излучателя и отражателя.

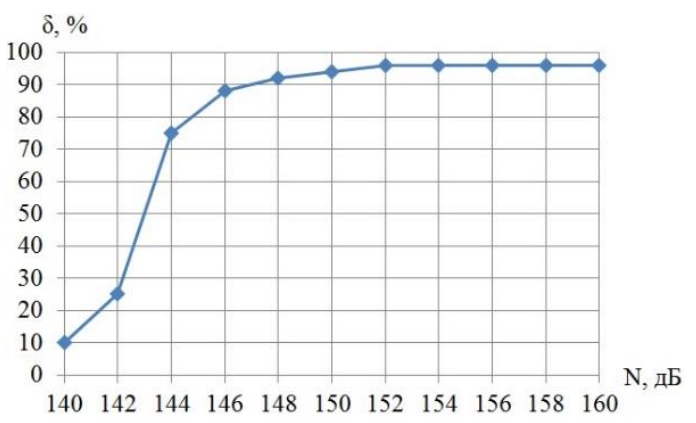

Рис. 16. Отношение эффективности ультразвуковой коагуляции к уровню звукового давления для дискового излучателя

Fig. 16. Dependence of the efficiency of ultrasonic coagulation on the level of sound pressure for disk radiator

Из полученных результатов следует возможность обеспечения эффективности коагуляции, равной $96 \%$, для дискового излучателя (в сравнении с 83 \% для излучателя поршневого типа).

Таким образом, эффективность коагуляции при помощи дискового излучателя, создающего акустические потоки вихревого типа, выше, чем при использовании излучателя поршневого типа, на $13 \%$. Так как условия проведения экспериментов были одинаковые, то указанное повышение может быть обусловлено только созданием вихревых акустических течений. При их формировании создаются условия для дрейфа частиц под действием центробежных сил из центральной области вихря к его периферии. В результате обеспечивается локальное повышение концентрации частиц в периферийной области вихря, повышающее вероятность столкновения, а, следовательно, и коагуляции частиц под действием УЗ колебаний.

Проанализировав зависимость на рис. 16, можно сделать вывод, что эффективность коагуляции изгибно-колеблющегося дискового излучателя достигает своего максимума при более низких уровнях звукового давления. Для изгибно-колеблющегося дискового излучателя видно, что, начиная с уровня звукового давления в 150 дБ, эффективность коагуляции достигает своего максимального значения и практически (не более 2 \%) перестает увеличиваться далее.

Также были проведены аналогичные эксперименты по исследованию эффективности процесса коагуляции дискового излучателя при различных концентрациях аэрозоля. Результаты исследований представлены на рис. 17.

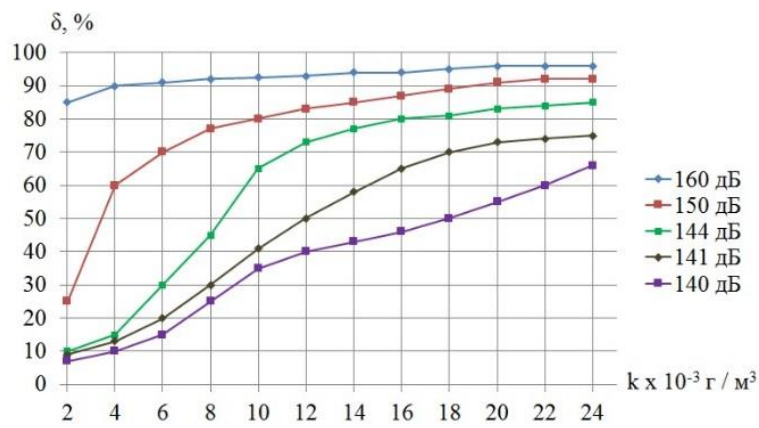

Pис. 17. Зависимость эффективности коагуляции от начальной кониентрации для дискового излучателя

Fig. 17. Dependence of coagulation efficiency on the initial concentration for a disk radiator

Из сравнения зависимостей на рис. 9 и 17 установлено, что при использовании дискового излучателя при малой концентрации частиц эффективность коагуляции значительно превышает эффективность излучателя поршневого типа. Эффективность коагуляции с использованием излучателя поршневого типа с увеличением концентрации частиц возрастает линейно и достигает своего максимального значения - $83 \%$ при значении концентрации $24 \cdot 10^{-3} \mathrm{\Gamma} / \mathrm{M}^{3}$, в то время как эффективность с использованием дискового излучателя достигла своего максимума - $96 \%$ - при концентрации уже $18 \cdot 10^{-3} \Gamma / \mathrm{M}^{3}$. Как уже упоминалось, 
оба излучателя работали при одинаковом уровне звукового давления, поэтому можно предположить, что такая разница связана с формированием вихревых акустических потоков дисковым изгибноколеблющимся излучателем.

При высоких начальных концентрациях аэрозоля ультразвуковое воздействие с формированием вихревых потоков дисковым излучателем целесообразно использовать на заключительной стадии процесса коагуляции, когда за счет воздействия ультразвуковых колебаний происходит снижение концентрации частиц, приводящее к квадратичному уменьшению вероятности агломерации частиц, а как следствие, и эффективности ультразвуковой коагуляции. То есть создание вихревых течений эффективно для сред с малой концентрацией частиц, потому что при характерных расстояниях между взвешенными частицами, намного превышающих их размер, силы гидродинамического взаимодействия частиц и амплитуда их колебательного движения оказываются недостаточными для сближения частиц на достаточное для их объединения расстояние. А за счет вихревых потоков концентрация локально повышается и за счет этого коагуляция имеет большую эффективность, чем без вихревых потоков [3, 12-14, 18, 19].

\section{Устройство для практической реализации предложенного способа УЗ коагуляции с использованием вихревых потоков}

Для практической реализации УЗ коагуляции с использованием вихревых потоков предложена практическая конструкция, в которой для создания высокоинтенсивных акустических полей используется пьезоэлектрическая колебательная система - 1 , состоящая из: пьезоэлектрического преобразователя электрических колебаний в механические - 2, концентратора-усилителя механических колебаний - 3, излучателя-рабочего инструмента в виде пластины (диска) 4 с плоской излучающей поверхностью. Перед излучающей поверхностью устанавливается отражающая ультразвуковые колебания поверхность - 5. Таким образом, ультразвуковая колебательная система устанавливается сверху коагуляционной камеры, формируемой между излучающей и отражающей поверхностями (рис. 18).

Через входной патрубок - 6, который располагается в центральной части отражающей поверхности, подается загрязненный частицам газ - 7, и он распространяется к периферическим областям отражающей поверхности в коагуляционной камере. Очищенный газ - 8 и скоагулированные (объединенные и увеличенные в размерах) частицы - 9 выводятся за пределы технологического объёма через выходные патрубки - 10. В процессе прохождения газа между двумя плоскостями (отражателем и излучателем) осуществляется ультразвуковое воздействие изгибно-колеблющейся пластиной, на плоской излучающей поверхности которой формируются максимумы и минимумы колебаний, чередующиеся последовательно на расстоянии, соответствующем половине длины волны изгибных колебаний в материале пла- стины на частоте не менее 22 кГц с интенсивностью не менее 160 дБ (рис. 18).

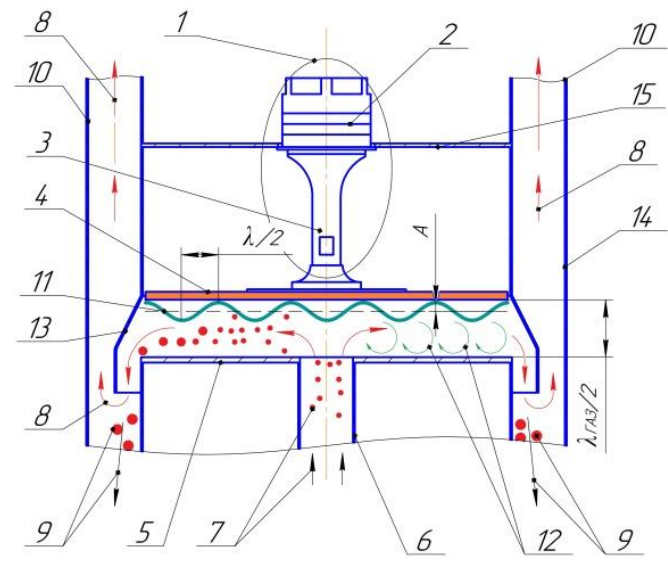

Рис. 18. Вариант реализации способа ультразвуковой коагуляичи

Fig. 18. Variant of implementation of the method of ultrasonic coagulation

Пластина с плоской излучающей поверхностью формует в зазоре между излучающей и отражающей поверхностями синусоидальное распределение амплитуд механических колебаний - 11. Благодаря созданию разнонаправленных колебательных движений на участках, отличающихся фазами на 180 градусов (условия создания разряжения и повышенного давления на области газа), образуются вихревые течения 12. Инородные частицы, попадая в эти вихревые потоки, более длительное время подвергаются УЗ воздействию, формируются условия повышения концентраций частиц на участках изменений направления их движения (рис. 18).

При этом частицы, по мере их продвижения к периферической области коагуляционной камеры, последовательно попадают в каждый последующий сформированный вихрь, увеличиваясь в размерах. После коагуляции частицы уносятся потоком воздуха к периферическим областям камеры, где сталкиваются с перегородкой - 13, которая служит для разделения очищенного воздуха и скоагулированных частиц. Корпус - 14 служит для обеспечения защиты расположенных внутри элементов от механических повреждений в процессе эксплуатации или хранения, а также для защиты от пыли, влаги и любых других внешних воздействий. За фланец - 15 крепится ультразвуковая колебательная система (рис. 18).

Таким образом, в предложенном устройстве для У3 коагуляции создание вихревых потоков за счет формирования УЗ колебаний в резонансном промежутке значительно увеличивает время и эффективность УЗ воздействия на загрязняющие газ частицы, а, следовательно, и саму эффективность процесса коагуляции частиц.

\section{Заключение}

В результате исследований выявлена возможность повышения эффективности УЗ коагуляции в резонансном слое с созданием вихревых потоков, предложен но- 
вый способ очистки газов от дисперсных частиц размером менее 2,5 мкм за счет увеличения времени УЗ воздействия на каждую частичку и создания зон локального увеличения концентрации тонкодисперсных и субмикронных частиц. Это обеспечивается путем формирования вихревых потоков между излучающей и отражающей поверхностями. Локальная зона повышенной концентрации частиц формируется в периферийной области вихря за счет дрейфа частиц под действием центробежных сил из центральной области вихря к его периферии. При этом излучающая поверхность выполняется в виде изгибно-колеблющегося диска.

В результате исследований установлено, что воздействие в режиме плоской стоячей волны, формируемой излучателем поршневого типа, обеспечивает предельное значение эффективности ультразвуковой коагуляции при уровне звукового давления 160 дБ, равное $83 \%$.

Показано, что для увеличения эффективности коагуляции необходимо создавать неоднородные ультразвуковые поля, способствующие формированию акустических течений вихревого типа. Экспериментально установлено, что в наибольшей степени вихревые потоки проявляются на расстояниях 7 мм $(\lambda / 2)$ и 14 мм ( $\lambda)$. При больших расстояниях значительное влияние вихревых акустических потоков на эффективность коагуляции отсутствует.

Сравнение эффективности коагуляции, обеспечиваемой поршневым и дисковым излучателями показало, что:

- при одинаковом уровне звукового давления, создаваемом излучателями обоих типов, дисковый излучатель обеспечивает более высокую эффективность коагуляции $(\delta=96 \%)$, чем излучатель поршневого типа $(\delta=83 \%)$ в газовом промежутке, равном одной длине волны УЗ колебаний в воздухе. Именно на таком расстоянии вихревые потоки проявляются наиболее сильно;

\section{СПИСОК ЛИТЕРАТУРЫ}

1. Очистка промышленных газов от пыли / В.Н. Ужов, А.Ю. Вальдберг, Б.И. Мягков, И.К. Решидов. - М.: Химия, 1981. - 386 с.

2. Ветошкин А.Г. Процессы и аппараты пылеочистки. - Пенза.: Изд-во Пенз. гос. ун-та, 2005. -210 с.

3. Numerical investigation of PM2.5 size enlargement by heterogeneous condensation for particulate abatement / F. Fan, S. Zhang, W. Wang, J. Yan, M. Su // Process Safety and Environmental Protection. - 2019. - V. 125. - P. 197-206.

4. Application of acoustic agglomeration to enhance air filtration efficiency in air-conditioning and mechanical ventilation (ACMV) system / Bing Feng Ng, Jin Wen Xiong, Man Pun Wan // PLOS ONE. - 2017. - V.12. - P. 1-26.

5. Оборудование для очистки газов от промышленных печей С.А. Козлова, И.М. Шалаев, О.В. Рева, А.В. Киселев. - Красноярск: СФУ, 2007. -156 с.

6. Ладыгичев М.Г., Бернер Г.Я. Зарубежное и отечественное оборудование для очистки газов справочное издание. - М.: Теплотехник, 2004. - 696 c.

7. Ультразвук. Газоочистка / В.Н. Хмелев, А.В. Шалунов, Р.Н. Голых, В.А. Нестеров. - Бийск: Изд-во Алт. гос. техн. унта, 2018. $-534 \mathrm{c}$.

8. Тимошенко В.И., Чернов Н.Н. Осаждение и осадконакопление промышленных дымов. - Ростов-на-Дону: Ростиздат, 2004. $224 \mathrm{c}$.
- формирование вихревых акустических потоков позволяет понизить (до 150 дБ) уровень звукового давления, необходимый для обеспечения максимальной эффективности коагуляции.

- формирование вихревых акустических течений наиболее целесообразно для аэрозолей малых концентраций. Так, для концентрации $18 \cdot 10^{-3} \Gamma / \mathrm{M}^{3}$ прирост эффективности, обеспечиваемый вихревыми потоками, составляет до 50 \%. При высоких концентрациях воздействие ультразвуковыми источниками, формирующими вихревые потоки, рационально использовать на заключительной стадии процесса коагуляции, когда за счет воздействия ультразвуковых колебаний происходит снижение концентрации частиц, приводящее к квадратичному уменьшению вероятности агломерации частиц, а как следствие, и эффективности ультразвуковой коагуляции.

Для практической реализации предложенного способа ультразвуковой коагуляции создано устройство, обеспечивающее формирование вихревых потоков за счет УЗ колебаний в резонансном промежутке и пригодное для практической реализации при создании устройств газоочистки от субмикронных загрязняющих частиц.

Полученные результаты могут быть использованы для повышения эффективности ультразвуковой коагуляции более крупных частиц микронного диапазона (5 мкм и более), поскольку такие частицы также будут вовлекаться в вихревое движение, а за счет большей массы такие частицы будут быстрее дрейфовать в направлении периферийной области вихря. Таким образом, для более крупных частиц вероятность столкновения, а, следовательно, и коагуляции под действием УЗ колебаний будет не ниже установленной в проведенных экспериментах.

Исследование выполнено за счет гранта Российского научного фонда (проект №19-19-00121).

9. Ткаченко Л.А. Динамика аэрозолей в акустическом волновом поле в трубе // Актуальные проблемы механики сплошной среды. - Казань: Институт механики и машиностроения Казанского НЦ РАН, 2016. - С. 100-114.

10. Particulate air pollution acute cardio respiratory hospital admissions and mortality among the elderly / J. Holonen, T. Lanki, T. Yli-Tuomi, P. Tiittanen, V. Kulmala, J. Pekkanen // Amrican Journal of Epidemilogy. - 2009. - V. 20. - P. 143-153.

11. Modal analysis and nonlinear characterization of an airborne power ultrasonic transducer with rectangular plate radiator / R.R. Andres, V.M. Acosta, M. Lucas, E. Riera // Ultrasonic. 2018. - V. 82. - P. 345-356.

12. Экспериментальное исследование процесса ультразвуковой коагуляции аэрозолей / В.Н. Хмелев, А.В. Шалунов, А.В. Боченков, В.А. Нестеров // Измерения, автоматизация и моделирование в промышленности и научных исследованиях (ИАМП-2019): Материалы XIV Всероссийской научнотехнической конференции студентов, аспирантов и молодых ученых с международным участием. - Бийск: Изд-во Алт. гос. техн. ун-та., 2019. - C. 243-248.

13. Experimental stand for the research of the process of ultrasonic coagulation of aerosols / V.N. Khmelev, A.V. Shalunov, A.S. Bochenkov, V.A. Nesterov, S.A. Terentiev, S.S. Zorin // 20th International Conference of Young Specialists on Micro/ Nanotechnologies and Electron Devices. EDM'2019: Conference Proceedings. - Novosibirsk: NSTU, 2019. - P. 221-226. 
14. Longitudinally oscillating ultrasonic emitter for influencing gasdispersed system / V.N. Khmelev, V.A. Nesterov, A.V. Shalunov, R.V. Barsukov, S.N. Tsyganok // Journal of Physics: Conference Series. - Krasnoyarsk: Krasnoyarsk Science and Technology City Hall of the Russian Union of Scientific and Engineering Associations, 2020. - V. 1679 (1). - P. 022008 (1-8). DOI: $10.1088 / 1742-6596 / 1679 / 2 / 022008$

15. Power ultrasonic transducer with extensive radiator for industrial processing / J.A. Gallego-Juarez, G. Rodriguez, V. Acosta, E. Riera // Ultrasonic Sonochemistry. -2010 . - V. 17. - P. 954-964

16. Gallego-Juarez A., Rodroguez-Corral G., Gaete-Garreton L. An ultrasonic transducer for high power applications in gases // Ultrasonic - 1998. - V. 16 (6). - P. 267-271.

17. Advances in the development of power ultrasonic technologies based on the stepped plate transducers / J.A. Gallego-Juarez,
G. Rodriguez, E. Riera, V.M. Acosta, F. Montoya, A. Blanco // 36th Annual Symposium. - Teddington, Middlesex, England, 2007. - P. 1-37.

18. Investigation of the influence of humidity on the ultrasonic agglomeration of submicron particles in diesel exhausts / E. RieraFranco de Sarabia, L. Elvira-Segura, I. Gonzalez-Gomez, J.J. Rodrigues-Maroto, R. Munoz-Bueno, J.L. Dorronsoro-Areal // Ultrasonic. - 2003. - V. 41. - P. 277-281.

19. Frequency comparative study of coal-fired fly ash acoustic agglomeration / J. Liu, J. Wang, G. Zhang, J. Zhou, K. Cen // Journal of environmental science. -2011 . - V. 23 (11). - P. 1845-1851.

Поступила 22.09.2021 г.

\section{Информация об авторах}

Хмелев В.Н., доктор технических наук, профессор, заместитель директора по научной работе Бийского технологического института (филиал) Алтайского государственного технического университета им И.И. Ползунова.

Шалунов A.B., доктор технических наук, заведующий кафедрой методов и средств измерений и автоматизации Бийского технологического института (филиал) Алтайского государственного технического университета им И.И. Ползунова.

Боченков $\boldsymbol{A . C .}$. аспирант Бийского технологического института (филиал) Алтайского государственного технического университета им И.И. Ползунова.

Нестеров В.A., кандидат технических наук, доцент кафедры методов и средств измерений и автоматизации Бийского технологического института (филиал) Алтайского государственного технического университета им И.И. Ползунова. 
UDC 681.2:681.722:534.838.7

\title{
DEVELOPMENT AND RESEARCH OF A NEW METHOD OF GAS CLEANING FROM PARTICLES LESS THAN 2.5 MICRON IN SIZE
}

\author{
Vladimir N. Khmelev 1 , \\ vnh@bti.secna.ru
}

\section{Andrey V. Shalunov ${ }^{1}$, shalunov@bti.secna.ru}

\author{
Alexander S. Bochenkov1, \\ sanya.bochankov@mail.ru \\ Viktor A. Nesterov ${ }^{1}$, \\ nva@u-sonic.ru
1 Biysk Technological Institute (branch) of Altay State Technical University named after I.I. Polzunov, 27, Trofimov street, Biysk, 659305, Russia.

\begin{abstract}
The relevance of the research is caused by the lack of effective methods and means of combating atmospheric pollution with particles less than 2,5 microns in size. The ultrasonic effect used for these purposes does not allow ensuring the degree of coarsening of dispersed particles sufficient for their sedimentation or capture by the existing gas cleaning equipment. Experimental studies show that even at the maximum sound pressure level (above which the reverse process - the dispersion of drops or the destruction of particle agglomerates - is initiated), the efficiency of ultrasonic coagulation is insufficient, especially at a low counting concentration of particles. This necessitates the identification of new physical effects and the development of new methods of ultrasonic exposure, providing an increase in the efficiency of coagulation of fine particles.

The main aim: development of a method for increasing the efficiency of ultrasonic coagulation of particles less than 2,5 microns in size due to emerging vortex acoustic flows in a thin air gap between the emitter and the reflector and a device for its implementation.

Results. The authors have proposed a new method and device for cleaning gases from dispersed particles less than 2,5 microns in size by increasing the time of ultrasonic action on each particle and creating zones of local increase in the concentration of fine and submicron particles. This is provided by the formation of vortex flows between the emitting (in the form of a flexural-vibrating disk) and the reflecting surfaces. A local zone of increased concentration of particles is formed in the peripheral region of the vortex, due to the drift of particles under the action of centrifugal particles from the central region of the vortex to its periphery. The proposed method provides the increase in efficiency from 13 to $50 \%$, depending on the initial concentration of dispersed particles.
\end{abstract}

\section{Key words:}

Ultrasonic, ultrasonic coagulation, radiator, vortex flows, aerosol.

The research was funded with the grant of the Russian Science Foundation (project no. 19-19-00121).

\section{REFERENCES}

1. Uzhov V.N., Valdberg A.Yu., Myagkov B.I., Reshidov I.K. Ochistka promyshlennykh gazov ot pyli [Cleaning industrial gases from dust]. Moscow, Khimiya Publ., 1981. 386 p.

2. Vetoshkin A.G. Protsessy $i$ apparaty pyleochistki [Dust cleaning processes and devices]. Penza, Penza State University Publ. House, 2005. $210 \mathrm{p}$.

3. Fan F., Zhang S., Wang W., Yan J., Su M. Numerical investigation of PM2.5 size enlargement by heterogeneous condensation for particulate abatement. Process Safety and Environmental Protection, 2019, vol. 10, pp. 614-628.

4. Bing Feng Ng, Jin Wen Xiong, Man Pun Wan. Application of acoustic agglomeration to enhance air filtration efficiency in airconditioning and mechanical ventilation (ACMV) system. PLOS ONE, 2017, vol. 12, pp. 1-26.

5. Kozlova S.A., Shalaev I.M., Reva O.V., Kiselev A.V Oborudovanie dlya ochistki gazov ot promyshlennykh pechey [Equipment for cleaning gases from industrial furnaces]. Krasnoyarsk, SFU, 2007. 156 p.

6. Ladygichev M.G., Berner G.Ya. Zarubezhnoe $i$ otechestvennoe oborudovanie dlya ochistki gazov. Spravochnoe izdaniye [Foreign and domestic gas purification equipment. Reference edition]. Moscow, Teplotekhnik Publ., 2004. 696 p.

7. Khmelev V.N., Shalunov A.V., Golykh R.N., Nesterov V.A Ultrazvuk. Gazoochistka [Ultrasound. Gas cleaning]. Biysk, Altai State Technical University Publ. House, 2018. 534 p.
8. Timoshenko V.I., Chernov N.N. Osazhdenie i osadkonakoplenie promyshlennykh dymov [Deposit and Sedimentation of Industrial Fumes]. Rostov-on-Don, Rostizdat Publ., 2004. 224 p.

9. Tkachenko L.A. Dinamika aerozoley v akusticheskom volnovom pole $\mathrm{v}$ trube [Dynamic of aerosols in the acoustic wave field in the tube]. Aktualnye problem mekhaniki sploshnoy sredy [Actual problems of continuum mechanics]. Kazan, Institute of Mechanics and Mechanical Engineering of the Kazan Scientific Center of the Russian Academy of Sciences Publ., 2016. pp. 100-114.

10. Holonen J., Lanki T., Yli-Tuomi T., Tiittanen P., Kulmala V., Pekkanen J. Particulate air pollution acute cardio respiratory hospital admissions and mortality among the elderly. American Journal of Epidemiology, 2009, vol. 20, pp. 143-153.

11. Andres R.R., Acosta V.M., Lucas M., Riera E. Modal analysis and nonlinear characterization of an airborne power ultrasonic transducer with rectangular plate radiator. Ultrasonic, 2018, vol. 82, pp. 345-356.

12. Khmelev V.N., Shalunov A.V., Bochenkov A.V., Nesterov V.A. Eksperimentalnoe issledovanie protsessa ultrazvukovoy koagulyatsii aerozoley [Experimental study of ultrasonic coagulation of aerosols]. Izmereniya, avtomatizatsiya $i$ modelirovanie $v$ promyshlennosti $i$ nauchnykh issledovaniyakh. Materialy XIV Vserossiyskoy nauchno-tekhnicheskoy konferentsii studentov, aspirantov $i$ molodykh uchenykh s mezhdunarodnym uchastiyem [Measurements, automation and modeling in industry and scientific research. Materials of the XIV All-Russian Scientific and Technical Conference of Students, Postgraduates 
and Young Scientists with International Participation]. Biysk, Altai State Technical University, 2019. pp. 243-248.

13. Khmelev V.N., Shalunov A.V., Bochenkov A.S., Nesterov V.A, Terentiev S.A., Zorin S.S. Experimental stand for the research of the process of ultrasonic coagulation of aerosols. $20^{\text {th }}$ International Conference of Young Specialists on Micro/Nanotechnologies and Electron Devices. EDM'2019: Conference Proceedings. Novosibirsk, NSTU, 2019. pp. 221-226.

14. Khmelev V.N., Nesterov V.A., Shalunov A.V., Barsukov R.V., Tsyganok S.N. Longitudinally oscillating ultrasonic emitter for influencing gas-dispersed system. Journal of Physics: Conference Series, 2020, vol. 1679 (1), pp. 022008 (1-8).

15. Gallego-Juarez J.A., Rodriguez G., Acosta V., Riera E. Power ultrasonic transducer with extensive radiator for industrial processing. Ultrasonic Sonochemistry, 2010, vol. 17, pp. 954-964.

16. Gallego-Juarez A., Rpdroguez-Corral G., Gaete-Garreton L. An ultrasonic transducer for high power applications in gases. Ultrasonic, 1998, vol. 16 (6), pp. 267-271.
17. Gallego-Juarez J.A., Rodriguez G., Riera E., Acosta V.M., Montoya F., Blanco A. Advances in the development of power ultrasonic technologies based on the stepped plate transducers. $36^{\text {th }}$ Annual Symposium. Teddington, Middlesex, England, 2007. pp. $1-37$.

18. Riera-Franco de Sarabia E., Elvira-Segura L., Gonzalez-Gomez I., Rodrigues-Maroto J.J., Munoz-Bueno R., Dorronsoro-Areal J.L. Investigation of the influence of humidity on the ultrasonic agglomeration of submicron particles in diesel exhausts. Ultrasonic, 2003, vol. 41, pp. 277-281.

19. Liu J., Wang J., Zhang G., Zhou J., Cen K. Frequency comparative study of coal-fired fly ash acoustic agglomeration. Journal of environmental science, 2011, vol. 23 (11), pp. 1845-1851.

Received: 22 September 2021.

\section{Information about the authors}

Vladimir N. Khmelev, Dr. Sc., professor, deputy director for academic affairs, Biysk Technological Institute (branch) of Altay State Technical University named after I.I. Polzunov.

Andrey V. Shalunov, Dr. Sc., head of the department, Biysk Technological Institute (branch) of Altay State Technical University named after I.I. Polzunov.

Alexander S. Bochenkov, postgraduate student, Biysk Technological Institute (branch) of Altay State Technical University named after I.I. Polzunov.

Viktor A. Nesterov, Cand. Sc., associate professor, Biysk Technological Institute (branch) of Altay State Technical University named after I.I. Polzunov. 